\title{
EVOLUCIÓN DEL ESTADO COLOMBIANO Y SU CAMINO HACIA LA PAZ
}

The evolution of the Colombian state and its path toward peace

\author{
Víctor Elías Guevara Flórez ${ }^{112}$ \\ $\underline{\text { Katherine Castañeda Ramírez }}^{113}$
}

Fecha de recepción: 12 de abril de 2018

Fecha de aceptación: 22 de mayo de 2018

SUMARIO: 1. Introducción; 2. Conflicto armado colombiano; 3. Análisis del acuerdo de paz entre las farc y el gobierno colombiano; 4. Conclusiones: políticas públicas necesarias para la implementación del acuerdo y la consolidación de la paz; 5. Conclusiones: retos del estado colombiano 6. Bibliografía

\footnotetext{
112 Abogado Universidad Libre, Filosofo Universidad de Cartagena, Especialista en Derecho Administrativo Universidad Libre, Candidato a Magister Universidad Federal de Integración Latinoamericana Unila- Brasil, Oficial mayor en el Tribunal Superior de Cartagena Sala Laboral. https://orcid.org/0000-0001-7990-5774 E-Mail: victorguevaraf27@gmail.com

113 Abogada de la Universidad de Cartagena. Asesora en Juridicaribe S.A.S. https://orcid.org/0000-0001-5718-2894 E-Mail: kthecastaeda9@gmail.com
} 
Víctor Elías Guevara Flórez, Katherine Castañeda Ramírez

\section{COMO SE CITA ESTE ARTÍCULO (APA 6)}

Guevara Flórez, Víctor Elías \& Castañeda Ramírez, Katherine (2018). Evolución del Estado colombiano y su camino hacia la paz. Revista Jurídica Mario Alario D’Filippo, Vol. X, №. 20, pág. $120-131$

\section{RESUMEN}

En este artículo se busca esbozar de manera crítica los desafíos que trae consigo la consolidación de la paz en Colombia, partiendo del surgimiento y fin del conflicto de la guerrilla de las farc-ep y el estado colombiano, lo anterior mediante una investigación exploratoria de fuentes secundarias de derecho que nos permiten el análisis histórico del origen del conflicto armado, y de las consecuencias derivadas de cerca de 60 años de violencia, teniendo como herramienta actual, un acuerdo de paz en el que se pactaron una serie de puntos de los que deviene la necesaria implementación de políticas públicas y estrategias de desarrollo, todas estos desafíos para el gobierno en Colombia.

\section{Palabras Claves}

Políticas públicas, estado, gobierno, conflicto armado, acuerdo de paz.

\section{ABSTRACT}

this article seeks to sketch critically the challenges that the consolidation of peace in Colombia brings, starting from the emergence and end of the guerrilla conflict of the farc-ep and the Colombian state, the foregoing through an exploratory investigation of sources secondary rights that allow us the historical analysis of the origin of the armed conflict, and the consequences arising from nearly 60 years of violence, having as a current tool, a peace agreement in which agreed a series of points from which it becomes the necessary implementation of public policies and development strategies, all these challenges for the government in Colombia.

\section{Key Words:}

Public policies, state, government, armed conflict, peace agreement. 


\section{INTRODUCCIÓN}

La historia del Estado colombiano ha estado colmada de un sin número de conflictos internos cuyas consecuencias ha marcado la vida social, económica y política del país, conflictos entre los cuales se encuentra la guerra librada durante cerca de 60 años entre el gobierno y las guerrillas. No obstante, a partir del acuerdo de paz logrado entre el gobierno nacional y las Fuerzas Armadas Revolucionarias de Colombia - FARC el año 2017, se avizora una significativa búsqueda por dejar atrás la historia de violencia en Colombia, en el camino por reconstruir el tejido social roto después de tantos años de conflicto.

Este acuerdo trae consigo grandes retos que vale la pena plantear y que nos lleva a cuestionarnos frente a la necesaria consecución de tareas gubernamentales para que el acuerdo por el cual las FARC EP dejo de existir como organización armada, sea la puerta hacia la consolidación de la paz estable en Colombia.

Estas tareas gubernamentales se traducen en la adopción de políticas públicas que atiendan no solo a las necesidades surgidas con ocasión del acuerdo de paz, sino de aquellas que dieron origen al conflicto de guerrillas en Colombia, a fin de que la historia de violencia no se repita.

Para ello, primero ha de tratarse las causas que llevaron al surgimiento de organizaciones armadas como las FARC EP y las acciones tendientes a cambiar dichas circunstancias. De otro lado, las consecuencias que ha dejado la lucha armada en el país y la forma en la cual se debe hacer frente a las mismas en especial en aquellas zonas de conflicto, y las contingencias que deben ser soportadas durante el post conflicto para que la paz se consolide, entre ellas, la existencia de grupos armados como el Ejército de Liberación Nacional - ELN y disidencias de las FARC, aunado a la cruenta batalla incesante contra el narcotráfico y los grupos ilegales. Todo ello, ligado a la polarización de la población frente a la aceptación del acuerdo de paz y el cambio de Gobierno por uno de corte abiertamente inconforme con el mismo, lo que trae consigo cambios de la agenda política que podrían significar crisis en la implementación del acuerdo y por ende del fin del conflicto armado.

\section{Conflicto armado colombiano}

La historia de Colombia está marcada por una prolongada violencia, extendida a lo largo del país y que ha afectado especialmente las zonas rurales llevando al desplazamiento de campesinos, aumento de la pobreza, proliferación del narcotráfico, entre otras consecuencias nefastas, en esta histórica confrontación han intervenido múltiples actores entre los cuales se encuentran la guerrilla de las FARC EP, uno de los protagonistas más antiguos según Pecaut (2008).

Los antecedentes de la conformación de esta y otras guerrillas en Colombia, han sido estudiados 
Víctor Elías Guevara Flórez, Katherine Castañeda Ramírez

por este autor, como una consecuencia de la política polarizada y hegemónica que sumió al país en una cruenta violencia en la disputa por el poder entre los partidos Liberal y Conservador, diferencias políticas que llevaron al derramamiento de sangre por todo el país, ello sumado a la influencia de las ideologías comunistas y la inspiración encontrada en la victoria de la revolución cubana, así como la lucha campesina por reivindicar los derechos a la tierra.

De tal forma que en Colombia surgieron grupos denominados autodefensas campesinas que más tarde conformarían entre otras guerrillas, la que hoy se conoce como FARC, grupo armado con influencia comunista, cuya búsqueda por la transformación del Estado se convirtió en una guerra de medio siglo y que hoy tiene fin con el acuerdo de paz celebrado en la Habana el 26 de agosto de 2012 .

Este acuerdo, a pesar de no ser la solución total de la violencia y desigualdad en Colombia, teniendo en cuenta que la historia de conflicto en Colombia no solo tiene como actor las guerrillas insurgentes, sino también grupos paramilitares, el mismo Estado a través de su fuerza armada, los actores del narcotráfico y bandas criminales, entre otros; si trae consigo un nuevo panorama de paz para el país que demanda grandes compromisos y cambios para su consolidación.

En ese sentido, el acuerdo de paz no es solo un documento mediante el cual se extingue un grupo armado, por más de destacar que se trata de la guerrilla más grande y antigua de Colombia, pues los puntos del acuerdo recaen en aspectos de incidencia nacional cuya puesta en marcha es necesaria a fin de borrar los desastres de la guerra.

\section{Análisis del acuerdo de paz entre las farc y el gobierno colombiano.}

El 24 de agosto de 2016 y tras diálogos sostenidos por delegados del gobierno nacional y de las FARC EP, se suscribió el acuerdo final para la terminación del conflicto con este grupo guerrillero, documento en el cual se plasmó la intención de construir paz estable y duradera a partir de la ejecución de seis puntos clave.

\section{a. Reforma rural integral:}

En este punto se plantea la necesidad de formalizar la propiedad sobre las tierras rurales, la promoción del acceso a la tierra a través de subsidios y facilitación de créditos priorizando a las mujeres como beneficiarias de los mismos, acceso que debe ir acompañado según lo pactado, de un soporte integral del estado a través de la capacitación, adecuación de suelos, recuperación de tierras y acceso a medios de producción.

Así también se planteó la creación de programas de desarrollo agrario con enfoque territorial, que permitan la explotación sostenible de los recursos y la superación de la pobreza, además del fomento de la economía solidaria y el cooperativismo. 


\section{b. Participación política:}

Este es uno de los puntos más importantes teniendo en cuenta que en el origen del conflicto influyó en gran medida el ejercicio de una política excluyente y monopolizada, razón por la cual el acceso a la participación de todos los actores sociales en la política nacional es un pilar fundamental para que la violencia no sea nuevamente un método para buscar las transformaciones que la sociedad demanda y que deben ser traducidas por quienes gobiernan. En ese sentido, en el acuerdo quedaron plasmados entre algunos de los puntos más relevantes, la garantía para el ejercicio de la oposición política, el fomento de la participación política de las comunidades en decisiones que los afectan, garantías para la movilización y la protesta social, reforma al sistema electoral y la creación de circunscripciones transitorias de paz.

\section{c. Fin del conflicto}

Con el propósito de consolidar el acuerdo celebrado, se pactó como compromiso la terminación de todas las acciones ofensivas entre las FARC EPS y el gobierno nacional, para lo cual se estableció como aspectos fundamentales, el cese bilateral del fuego, la dejación de armas y la reincorporación de los miembros de las FARC en la vida civil, política y económica, siendo este un proceso que debe ser integral y progresivo, para lo cual es necesaria la reconstrucción del tejido social y la reconciliación de los actores.

\section{d. Solución al problema de drogas ilícitas}

Partiendo de la base de que el narcotráfico ha impulsado el conflicto armado en Colombia, proporcionando nos solo recursos económicos para la financiación de la guerra sino también el control de las zonas campesinas a través de los cultivos ilícitos, problemática que hoy es aguda considerando que cerca de 60 mil familias colombianas se dedican exclusivamente a los cultivos ilegales y subsisten del producto de los mismos, es evidente que para la construcción de paz necesariamente se requiere la erradicación de esta problemática y en ese sentido, en el acuerdo de paz se pactó de un lado, la necesaria transición campesina hacia la sustitución de cultivos, objetivo frente al cual por ejemplo, se estableció la creación del Programa Nacional Integral de Sustitución de Cultivos llícitos, proyecto que va ligado a las acciones derivadas de la reforma rural integral consignada en el punto primero del acuerdo de paz, como lo es el acceso a la tierra y la facilitación de la producción agrícola mediante la asistencia y estimulo del Estado y el mejoramiento de infraestructura vial en las zonas rurales. $Y$ de otro, la atención al problema del consumo de drogas frente a lo cual ha de implementarse políticas de prevención y atención. Todo esto ligado al fortalecimiento de las estrategias en la lucha contra la criminalidad y el narcotráfico.

\section{e. Víctimas y justicia transicional}

Se busca corresponder a los principios de verdad, justicia, reparación y no repetición, garantizando a las víctimas del conflicto armado su derecho al esclarecimiento de la verdad, para 
tal fin se crearon distintos organismos entre los cuales se encuentra, la Comisión para el esclarecimiento de la verdad, la convivencia y la no repetición; y la Unidad Especial para la búsqueda de personas dadas por desaparecidas.

Así también se creó la Jurisdicción Especial para la Paz (JEP), como mecanismo encargado de garantizar la justicia, y que solo procede frente a conductas cometidas antes de la celebración del acuerdo, sin dejar de mencionar la importancia de los procesos de reparación y garantía de no repetición.

\section{f. Implementación, verificación y refrendación del acuerdo.}

Con el fin de hacer seguimiento a los componentes del Acuerdo Final y verificar su cumplimiento, además de resolver las diferencias que sobre él surjan, se acordó la creación de la Comisión de Implementación, Seguimiento y Verificación del Acuerdo Final de Paz y de Resolución de Diferencias (CSVR) la cual tendrá una duración hasta de diez años, con un periodo inicial de funcionamiento hasta enero de 2019.

Este organismo es el encargado de promover la integración de las poblaciones, comunidades y territorios, con especial énfasis en las zonas más afectadas por el conflicto, la pobreza y la marginalidad y debe crear los mecanismos que permitan la participación ciudadana con incidencia efectiva en las decisiones de las autoridades públicas, en la definición de prioridades y en la formulación de proyectos.

\section{Conclusiones: políticas públicas necesarias para la implementación del acuerdo y la consolidación de la paz.}

A partir de los acuerdos logrados en la Habana se plantean grandes compromisos para el gobierno nacional tendientes a consolidar la paz en el país, para lo cual se requiere la implementación de políticas públicas que desarrollen cada uno de los aspectos planteados como puntos del acuerdo, no obstante en esta oportunidad, se hace especial énfasis a dos aspectos puntuales como lo es la necesaria implementación de políticas públicas de acceso y explotación de la tierra y las políticas de desplazamiento y atención y reparación de las víctimas, considerando que de un lado, una de los principales causas del conflicto en Colombia obedece a la inequitativa distribución de la tierra y la extrema pobreza de las zonas rurales y de otro, entre sus consecuencias más nefastas se encuentra el despojo de los campesinos y el desplazamiento forzado.

En ese sentido, el Estado juega un papel importante ajeno a los intereses del gobierno o de las FARC, pues debe tener la capacidad de suministrar los medios posibles para la consecución de los fines del acuerdo, esta capacidad entendida desde la definición de O’Donnel (2015, pg.59), como la habilidad para brindar bienes públicos y para respaldar a la economía con un marco legal fiable. De modo que no se pretenda que con ocasión de los acuerdos el Estado colombiano se convierta en un Estado benefactor y proporcione todo lo que la sociedad necesite, pero sí que se asuma la 
Víctor Elías Guevara Flórez, Katherine Castañeda Ramírez

ejecución de políticas públicas integrales que permitan crear un orden social más justo y equitativo, como factor fundamental para la consolidación de la paz.

Frente a lo anterior, es preciso aclarar que se adopta como política pública la definición dada por Villanueva (2012, pág. 5), es decir, las acciones de gobierno, que tienen como propósito realizar objetivos de interés público con eficacia y aun eficiencia.

La realización de estas acciones debe ser prioridad en la agenda política nacional, a fin de que se afronten los problemas nacionales que obstaculizan la consolidación de la paz, ello considerando lo esbozado por Oszlak (2011, pag.9), cuando refiere que los intereses que resultan representados y satisfechos por el Estado dependen, del contenido de la agenda de cuestiones socialmente problematizadas cuya vigencia sostiene, y resolución influye, la particular estructura de dominación impuesta en la respectiva sociedad.

Ahora bien, para la implementación de estas políticas el Estado ha de tener claridad de los problemas de interés que requieren la implementación de acciones de gobierno, para lo cual es necesaria una constante comunicación y lectura de las necesidades sociales de cara a la construcción de paz, sin ignorar la constancia y coherencia resaltada por Villanueva (2012), cuando expresa que las políticas públicas deben ser un conjunto de acciones intencionales y causales, orientadas a la realización de un objetivo de interés / beneficio público, cuyos lineamientos de acción, agentes, instrumentos, procedimientos y recursos se reproducen en el tiempo de manera constante y coherente. Así mismo, cuando precisa que es la estructura estable de sus acciones durante un cierto tiempo lo que hace específico y distintivo a ese conjunto de acciones de gobierno que llamamos política pública." (pág. 17.)

\section{a. Políticas públicas de acceso y explotación de la tierra}

El acuerdo de paz introduce como pilar fundamental la necesidad de una reforma agraria integral para la cual es necesaria la implementación de acciones de gobierno tendientes a incrementar las posibilidades de acceso a la tierra que permitan una distribución más igualitaria de la misma, así también, es necesario implementar políticas de recuperación de tierras y retorno de las familias campesinas despojadas durante el conflicto, sin olvidar que no basta el acceso a la tierra, como quiera que el problema agrario en Colombia radica además en las grandes dificultades de los pequeños y medianos productores para el sostenimiento de sus cultivos, por lo que se requieren políticas de asistencia por parte del Estado mediante el acompañamiento constante a través de la capacitación y soporte económico.

Así las cosas, como políticas concretas que devienen del acuerdo de paz se plantean: 
Víctor Elías Guevara Flórez, Katherine Castañeda Ramírez

- La creación de un fondo de tierras de distribución gratuita de tierras provenientes de: extinción de dominio (predios dedicados a actividades ilícitas como el narcotráfico), baldíos recuperados que habían sido indebidamente apropiados, tierras provenientes de actualización y delimitación de zonas de reserva forestal, tierras inexplotadas, tierras adquiridas o expropiadas por motivos de interés social y utilidad pública para promover el acceso a la propiedad rural, con la correspondiente indemnización y tierras donadas.

- Subsidio integral para la compra de insumos agrarios: en zonas priorizadas y con especial énfasis en las mujeres campesinas.

- Crédito especial para compra se insumos agrarios.

- Creación de Programas de Desarrollo Agrario Integral con Enfoque Territorial (PDET) para el acompañamiento en vivienda, asistencia técnica, capacitación, adecuación de tierras y recuperación de suelos, proyectos productivos, comercialización y acceso a medios de producción.

- Plan Nacional de Vías terciarias para promover integración regional y el acceso a servicios sociales y mercados.

- Plan Nacional de Riego y Drenaje para la economía campesina, familiar y comunitaria: para impulsar producción agrícola familiar y economía campesina.

- Plan Nacional de Electrificación Rural y Plan Nacional de Conectividad Rural.

- Implementación de programas de fomento a la economía solidaria y cooperativa.

\section{b. Políticas públicas de atención y reparación de víctimas y retorno de personas desplazadas}

Tras la celebración del acuerdo de paz y el fin de las hostilidades entre el Gobierno y las FARC, y con el propósito de reconstruir el tejido social roto tras los años de guerra en los cuales se vieron afectadas miles de familias colombianas, resulta imperioso fomentar acciones tendientes a la reparación de las víctimas, en un camino de esclarecimiento de la verdad en cuanto a los responsables de los perjuicios causados, sumado al resarcimiento de los mismos y el derecho de obtener justicia sin dejar de lado la reconciliación de los actores.

En ese sentido, las políticas cuya implementación se considera pertinente a partir de los acuerdos de paz celebrados son:

- La creación de una comisión para el esclarecimiento de la verdad, la convivencia y la no repetición: a fin de reconocer a las víctimas y los hechos acontecidos integrando para ello a todos los miembros de la sociedad, con especial énfasis en el reconocimiento de la vulneración de los derechos de la mujer.

- La creación de la Unidad Especial para la búsqueda de personas dadas por desaparecidas, mecanismo extrajudicial y de carácter humanitario, en el que tiene especial incidencia la 
Víctor Elías Guevara Flórez, Katherine Castañeda Ramírez

implementación de las políticas interdisciplinares desarrolladas por Villanueva (2012), como quiera que se requiere la coordinación de multiplex instituciones y actores, pues se requiere la búsqueda, identificación y entrega de restos de personas desaparecidas a sus familiares para lo cual participan varias instituciones de distintas disciplinas, entre ellas, el Instituto Nacional de Medicina Legal, organizaciones de víctimas y defensoras de derechos humanos, así como la Comisión de la Verdad.

- Políticas para la Implementación de la Jurisdicción especial para la Paz como el mecanismo encargado de garantizar justicia en el marco de los acuerdos y que tendrá sólo jurisdicción para conductas cometidas antes de la entrada en vigor de los acuerdos. Para lo cual se debe determinar claramente los delitos no amnistiables o indultables, pues la justicia incluye a quienes financiaron o apoyaron a los grupos en conflicto, aunque no pertenezcan a ellos.

- Acciones tendientes a garantizar el pleno goce de los derechos, la protesta pacífica, defensa de los derechos humanos o el liderazgo de grupos de la sociedad civil.

- Implementación de programas de Rehabilitación psicosocial, a fin de promover la recuperación de los procesos comunitarios de tejido social y el acompañamiento a prácticas sociales, culturales y artísticas como mecanismos de reparación.

\section{Conclusiones: retos del estado colombiano}

El Estado entendido en este caso como lo presenta O'Donnel (2015, pg.29), el aparato estatal conformado por un conjunto de instituciones políticas, y de relaciones sociales que establecen cierto orden y fuerza coercitiva , o analizado desde su manifestación material por Oszlak (2007, pg.2), y definida como un conjunto interdependiente de instituciones que conforman el aparato en el que se condensa el poder y los recursos de la dominación política, funge un papel determinante de cara a los desafíos que surgen de los compromisos pactados por el gobierno y las FARC EP y en el camino de ejecutarlos a fin de que estos no se disuelvan.

Retos estos como lograr el consenso político y social en torno a la implementación de los acuerdos y la legitimidad de los mismos, considerando que a pesar de tratarse de un acuerdo que pone fin a una guerra de cerca de medio siglo, en la actualidad continua siendo marcada la división ideológica entre quienes defienden y rechazan lo pactado, siendo en este punto una sociedad dividida en relación a los medios por los cuales se pretende la paz nacional, circunstancia que se evidenció claramente tras la votación del plebiscito para la aceptación del acuerdo, en el cual se obtuvo una mayoritaria negativa al acuerdo de paz, sin ser menos que rescatable que aquellas poblaciones en las cuales se evidenció mayor aceptación de la implementación del mismo, fueron zonas territoriales en las cuales el conflicto ha dejado mayores consecuencias.

De igual forma es un reto materializar en debida forma el derecho a la paz, como bien lo plantea 
Víctor Elías Guevara Flórez, Katherine Castañeda Ramírez

Mercado (2018, p. 196), "El mentado artículo 22 de la carta del 91 sobre la paz como derecho y deber de obligatorio cumplimiento es ilusorio, ya que como derecho ¿Quién lo ejerce? y como obligación ¿a quién se reclama? Es en realidad una confesión de un buen deseo y nada más dado que la paz es un problema político y no jurídico.

Así también, es un reto del Estado hacer frente a las dificultades sobrevinientes de cara al acuerdo de paz, como lo es la lucha que continúa en relación a los grupos disidentes de las FARC y la implementación de verdaderos canales de participación que garanticen el ejercicio de derechos políticos.

A fin de que tales aspectos sean asumidos logrando que en modo alguno amenacen la consolidación de los acuerdos de paz, ha de tenerse en cuenta la necesidad de que el Estado reoriente el orden establecido hacia uno que proporcione condiciones más iguales entre los ciudadanos.

Ello por cuanto se comparte la postura a partir de la cual el orden impuesto por el Estado a través de canales como el sistema legal, reproduce de una u otro forma relaciones de poder y en tal sentido no se trata de un orden igualitario, generando inevitablemente problemas sociales.

De acuerdo a De León \& Ballestas (2017, p. 48) una sociedad en paz puede focalizar sus esfuerzos en el cierre de brechas y puede invertir recursos en mejorar la cobertura y calidad de su sistema educativo. Una sociedad equitativa en donde todos los habitantes gozan de los mismos derechos y oportunidades permite la convivencia pacífica y facilita las condiciones de formación en capital humano.

En relación a ello el Estado colombiano debe replantear el orden establecido en medio del cual se gestó el conflicto en Colombia, pues se observa por ejemplo, que tras la promulgación de leyes de apertura económica que ignoraron las carencias del campesinado nacional y las dificultades para la producción de bienes agrícolas, se produjeron las mayores crisis del campo en el país llevando incluso a la conformación de grupos campesinos por la defensa de sus derechos que posteriormente también tendrían incidencia en la conformación de los grupos armados.

Así las cosas, el orden impuesto debe propender por garantizar la igualdad de los ciudadanos pues tal como lo resume O'Donnel (ANO, pg.31), la igualdad garantizada a todos los miembros de una nación en términos de ciudadanía es crucial para el ejercicio de derechos políticos que connota el funcionamiento de la democracia.

De otro lado, frente a estos retos el Estado cumple un papel fundamental debido a que su capacidad para afrontar las barreras que dificultan la consolidación de la paz es determinante. De 
Víctor Elías Guevara Flórez, Katherine Castañeda Ramírez

este modo, tal como plantea O’Donnell (2015) el Estado ha de intervenir con toda su capacidad fiscal en la distribución entre los grupos de la sociedad, a fin de hacer frente a la alta concentración de poder político y económico

Pero así también se requiere capacidad administrativa, burocrática, organizacional y coercitiva, para proporcionar mayores bienes que permitan la superación de la pobreza sobre todo en las zonas rurales del país, suministrando por ejemplo infraestructura para el acceso a las comunidades, de sistemas de transporte, educación y salud, a través de políticas integrales y sin dejar de lado la necesidad de contener coercitivamente los actores que siguen generando conflicto a través de la guerra y las actividades delictivas.

\section{REFERENCIAS BIBLIOGRÁFICAS}

ACUERDOS DE PAZ firmados entre gobierno nacional de Colombia y la guerrilla de las FARC, 24/08/2016.

AZAMBUJA, Darcy. Introdução à Ciência Política. São Paulo: Editora Globo, 2011. Cap. 10, 11,14, 15 e 17. (Acervo Biunila).

DE LEÓN Vargas, Georgina \& BALLESTAS León, Damary Margarita (2017). Derecho y economía: Fórmula para la superación de la pobreza extrema y la construcción de paz en Colombia. Revista Jurídica Mario Alario D’Filippo, IX (18), pág. 41-54.

KALACH, G. (2016). Las comisiones de la verdad en Colombia (2016). Revista Jurídica Mario Alario D'Filippo. VIII, (16): 106-124. Recuperado de DOI: https://doi.org/10.32997/2256-2796-vol.8num.16-2016-1534

MARQUES, Eduardo. As políticas públicas na ciência política. IN: MARQUES, Eduardo e FARIA, Carlos Aurélio Pimenta de. A política pública como campo multidisciplinar. Pg.23-46.

MERCADO, D. (2018). La Antinomia Entre Justicia Y Política En Colombia. En: Carrillo, Y., Mercado, D. y Luna, F. (Coords.) La Teoría Constitucional Colombiana Frente Al Constitucionalismo Global. Bogotá. Ediciones Nueva Jurídica. 2018. Págs.: 193-230

OSZLAK, C. Formación Histórica del Estado En América Latina: Elementos Teórico - Metodológicos para su estudio. IN: ACUÑA, Carlos, H. (comp.) Lecturas sobre el Estado y las políticas públicas: Retomando el debate de ayer para fortalecer el actual, Proyecto de Modernización del Estado, Jefatura de Gabinete de Ministros. Buenos Aires, 2011. 
O’DONNELL, G. Estado, democratización y ciudadanía. IN: CHUDNOVOSKY, M. (comp.) Capacidades Estatales. Diez textos fundamentales. Argentina: Editor Caf, 2015.

Salgado González, A. (2017) Constitución y Derechos Humanos. Revista Jurídica Mario Alario D'Filippo, 9 (18): 21-30. Recuperado de DOI: https://doi.org/10.32997/2256-2796-vol.9-num.18$\underline{2017-2051}$

SECCHI, L. Políticas Públicas: conceitos, esquema de análise, casos práticos. 2. Ed. São Paulo: Cengage Learning, 2015. 1-Introdução: percebendo as políticas públicas pág. 1-21. (Acervo Biunila)

PÉCAUT, Daniel. Las FARC: fuentes de su longevidad y de la conservación de su cohesión. Bogotá. 2008. Pag 26.

VILLANUEVA, R. Política Pública Una visión panorámica. Programa de las Naciones Unidas para el Desarrollo (PNUD - Bolivia), 2012. Cap. 2 El nacimiento de la disciplina de Política Pública y su recepción en América Latina (da pág. 6 até 16 do livro). 